Hospital Robetto del Río.

Cátedra da Pediatria

Prof, A, Scroggie.

\title{
TUBERCULOSIS RENAL DEL NIÑO
}

\section{Pox los Dres. LUIS CID y MARIO GONZALEZ}

En el presente trabajo damos a conocer la observación de un caso de tuberculosis renal en un niño de 2 años 6 meses. Ofrece este paciente un doble interés; sabemos que a esta edud es excepcional este tipo de lesión; por lo general, si se encuer:tra es una de las tantas manifestaciones de la tubercuiosis miliar. De abí que en nuestro paciente debamos suponer an grado sorprendente de defensas contra la tuberculosis, pues los caracteres de la lesión que describiremos (esclerosis, etc.). sólo es dable encontrarlo en individuos llegados a la edad adulta. En segupdo lugar, es éste uno de los pocos casos de curación total que se registra en la literatura, americana y europea. Decimos curación, pues hemos controlado a nuestro lactante. dos años después de operado en fcrma brillante por el prof. Bisquertt y $D_{r}$. Cantín, y se encuentra comp'etamente sano.

La tuberculosis urogenital es relativamente rara en el lactante y en el niño mayor. De cada sesenta pielitis crónica. una es tubèrculosis renal caseosa. La tuberculosis tenal se puede dividir en aguda y crónica.

\section{A) Tubercalosis renal miliar agada.}

Forma parte de la tuberculosis bematógena generalizada, no responde a ningún tratamiento y lleva un curso fatal. En el riñón, la tuberculosis miliar tiene predominantemente una localización cortical. El órgano muestra numerosos tubérculós blanco-grisáceos, netamente delimitados y en su pe- 
riferia presentan una zona de marcada congestión. Microscópicamente se puede apreciar que la embolia bacilar se localiza principalmente en la base de las pirámides, sin excluir por supuesto, las lesiones, aunque menos intensa en los glomérulos y en la medula. El progreso de la lesión se produce por caseificación, siendo muy raro que el paciente sobreviva el tiempo suficiente para que se produzca nn absceso tuberculoso.

\section{B) Tuberculosis renal crónica.}

M. Campbeil la clasifica en la siguiente forma:

I. Ulcerativa (úlcero cavernosa y pionefrótica).

2. Difusa nódulo esclerótica.

Esencialmente estas formas requieren el mismo tratamicrito, pe:o necesariamente no presentan el mismo cuadro clínico. Al referirse a tuberculosis renal, es la primera forma la que se tiene en cuenta.

\section{Etiologia.}

Frecuencia. - La forma miliar aguda es nás frecuente en el niño que en el adulto, en cambio. la tubercalosis renal caseosa crónica es a la inversa: sabemos que el desarrollo de una lesión crónica indica un grado mayor de inmunidad, de resistencia. De 12,082 autopsias de niñcs registrados por Campbell, sólo cinco eran tuberculosis renal crónica ( 1 x 240 ). Kapsammer, de 20,770 , encuentra 196. Vignatd y Thevenot, de 400 autopsias sólo encontraron tuberculosis renal, en forma miliar, en 82.

Edad. - En el feto y en el recién nacido han sido des. crito casos de tuberculcsis miliar. Vignard y Thevenot sólo encuentran cuatro meses de tuberculosis renal quirúrgica por debajo de los tres años. Eckehorn cita sólo un caso por debajo de los cinco años. Wildbolz sólo encuentra cuatro menores de diez años en 532 operados de tuberculosis renal.

Bardenhauer operó un riñón tuberculoso en un niño de tres meses, y Campbell, uno de veinticinco meses. De esto se deduce que la tuberculosis renal quirúrgica es rara en la infancia, aumenta rápidamente a medida que se avanza en edad. La tuberculosis genital asociada es también mucho menos común en el niño que en el adulto. Bumpus y Thompson la encontraror en dos de 606 casos de tuberculosis genital. 
Sexo y lado comprometido. - Según las estadísticas, de Vignard $\mathrm{y}$ Thevenot, es más frecuente en los niños (quince niñas y veintiún niños) y lo mismo encuentra Eckehorn. En lcs adultos se compromete más el lado derecho, proporción cinco por cuatro. Campbell cita siete derechas, cinco izquierdas y seis bilaterales. Pandorff encuentra lesiones bilaterales. en el $70 \%$ de los casos y lo atribuye a falta de diagnóstico precoz.

Tendencia familiar e inmunidad. - En los niños mayores, generalmente la tuberculosis es adquirida por el contacto con un familiar afectado de tuberculosis. En los niños afectados de tuberculosis renal, el compromiso mediastinal o pulmonar es demostrable clínica o radiológicamente en el $50 \%$ de los casos y en la necropsia en la casi totalidad. El hecho de que un niño haga una tuberculosis renal quirúrgica, indica que este niño presenta un grado de inmunidad muy superior âl corriente.

Factores predisponentes. - Desde luego, el principal, es cohabitar con tuberculosos pulmonares abiertos, y además. todos los otros factores que predisponen a infecciones agudas - crónicas de otra naturaleza (malformaciones congénitas, etc.).

Causa directa. - Siempre es secundaria a una infección tuberculosa de otro órgano, ya sea entérica, ganglionar o pulmonar, etc. Sin embargo, a veces la lesión inicial. no puede ser demostrada, aun en la necropsia.

Algunos autores, pensando que la leche es el alimento base de la infancia, han creido que es el bacilo bovino el principal causante; sin embargo, en estudios realizados por Griffith no fué encontrado en ningún caso.

Es frecuente no encontrar el bacilo de Koch en la orina y muchas veces la infección secundaria hace más difícil la búsqueda. Con las técnicas actualmente en uso, se les encuentra en un 80 a $90 \%$ de los casos. En las tuberculosis miliares es más frecuente su aparición en la orina que en los otros tipos de lesiones.

Vías de invasión. - La más frecuente es la vía hematógena.

La transmisión linfática es muy improbable; sólo puede aceptarse como forma mixta, linfohematógena; después de las experiencias de Wildbolz (inoculación de la vaina periurete. ral). La infección renal ascendente es menos probable aún que la anterior, serviría para explícar la propagación renal de 
una afección genital, siempre que se produzca un ectasis urinario crónico (experiencias de Wildbolz).

Fatclogía. - Algunos autores explican la lesión unilateral por una embolía bacteriana. Otros creen que la infección renal es bilateral al principio y luego se localiza en el locus minoris resistenciae. El desarrollo de la tuberculosis defende por una parte de la virulencia del bacilo y por otra de la resistencia local del paciente.

De preferencia empieza la lesión tuberculosa en la medula, y Eckehorn la explica por la gran vascularización de esta porción en donde la siembra hematógena es más probable; Pels-Leusden lo han demostrado en trabajo experimental. Medlar ha demostrado experimentalmente también que lericnes renales tubercuiosas pequeñas pueden no producir ningún sintoma y cutar espontáneamente, especialmente si eran corticales: esto no sucede en las lesiones quirúrgicas, que tiende siempre a progresar $\mathrm{y}$ a invadir otros órganos.

Cuando la lesión tuberculosa sobrepasa la resistencia local, ella se disemina hacia el proceso papilar, dentro del cual cl segmento afectado drena normalmente, y así en la orina tenemos baciluria y leucocitos. Jamás pasan los bacilos de Koch a través de un riñón sano (trabajos experimentales de Medlar, Helmholz, Spetzer, Williams, Burdick, Cronec).

Histclógicamente, las lesiones renales muestran una infiltración linfocitaria junto con células epitelioídeas y gigantes: el proceso puede curar por esclerosis o progresar con necrosis central, alrededor del cual se desarrolla una gran infiltración polimorfonuclear. Este absceso se vacia al interior de uno o más túbulos colectores, en el lumen de los cuales se encuentran bacilcs, pus y a veces glóbulos tojos; así tenemos cómo en las infecciones no tuberculosas, que el pus urinario se forma primero en el parénquima renal que en la pelvis. Dentto del riñón la infección tuberculosa se disemina por los linfáticos intrarrenales, sin descartar la posibilidad de la propagación for vía hematógena o tubular (Crabtree). Las conexiones de los linfáticos intrarrenales con los de la cápsula y la grasa perirrenal explica la presencia de perinefritis en las tuberculosis renales crónicas.

Las múltiples formas de tuberculosis renal crónica pueden ser consideradas como diferentes estados de una misma lesión.

Tuberculosis ulcerativa. — Podemos distinguir una forma precoz, en que la lesión puede estar confinada a una papila o a una porción de la medula; así Schneider describió 
una papilitis ulcerativa en un niño de catorce años y Campbell operó un niño de 25 meses que presentaba una lesión ulcerativa en una pirámide. Si la lesión está capsulada, la pelvis y la orina excretada son normales; cuando la pelvis es invadida, la orina contiene pus, sangre y B. K. El proceso tuberculoso se extiende a la pelvis y al utéter y el resto del órgano, aunque aparentemente sano, muestra microscópicamente propagación de la infección a las zonas corticales y subcorticales. La forma avturadz o tuberculosis caseo-cavernosa reve!a áreas esparcidas en gran número que contienen casum y a veces presentan cierto grado de reparación esclerótica. $\mathbf{M u}$ chas de estas lesiones están en comunicación con la pelvis, la cual ptesenta cierto grado de induración, así como también la vejiga. Esta es la forma más comúnmente encontrada en la operación.

El grade de destrucción del parénquima renal depende del drenaje urinario; si éste es deficiente, la destrucción renal cs mayor y más rápida, pues se agrega al factor infección un factor mecanico; a feces, cuando hay un buen drenaje, las cavidades pueden vaciarse totalmente. dejando una úlcera que puede llenarse de granulaciones y aun epiteliarizarse. Se comprende que esto es raro, pues es frecuente que se produzcan cstrecheces ureterales o extraureterales, por cicatrices retráctiles, originándose una hidronefrosis, presentándose el riñón aumentado de tamaño y lobulado.

Hay, además, una lipofibromatosis o una estlerosis perinefrítica y al tratar de separar la cápsula se desprende con irozos de corteza, tan firmemente están adheridos.

Un grado más avanzado de la lesión descrita constituve la pionefrosis tuberculosa. Aquí el riñón está aumentado ana y media a tres veces su tamaño, la superficie es lobulada con cicatrices y grupos de pequeñes tubérculos. Otras veces el ótgano es más pequeño que lo normal, debido a esclerosis $y$ retracción del órgano. Ocasionalmẹnte, el órgano está convertido en una bolsa hidronefrótica de paredes delgadas. El contenido del absceso es generalmente blanco o blanco amarillento, inodoro, espeso, lleno con pus cremoso, caseoso, a menudo con macilla, pero ocasionalmente con cal o calcificado (riñón cementado o mastic). A veces la masa pionefrótica se cierra o queda aislada por obstrucción areteral (autonefrectomia), desaparece la_piuria y los sintomas urinarios (polaquiuria). Radiológicamente un riñón así puede sugerir un gran cálculo renal, pero de sombra irregular. La autonefrectomía, a pesar de la mejoría subjetiva aparente, no trae incluida una 
mejoria local, pues los B. K. siguen en actividad y en cualquier momento puede venir una diseminación.

\section{Tuberculosis nódulo esclerótica.}

Alrededor del $1 \%$ de las tuberculosis renales asumen esta forma. La evolución del proceso es lenta y evidencia una alta resistencia a la infección; en la orina se encuentran escasos bacilos tuberculosos. El riñón está pequeño a causa de la contractura escletótica, pero a veces se suelen palpar por la intensa perinefritis. El tiñón toma un aspecto tosco, con masas nodulares de cicatriz.

Respecto al riñón sano, muchas veces puéde presentar una nefritis tóxica. Esto resulta dé lesiones causadas por tuberculotoxinas elaboradas en el órgano enfermo o en otros focos activos. El parénquima de este órgano es normal o puede presentar un discreto grado de tumefacción turbia, la orina de este lado puede contener pequeña cantidad de albúmina células del epitelio tubular, cilindros granulosos y a veces glóbulos rojos. La cilindruria puede aparecer en grandes descargas. Esta lesión desaparece con la extirpación del riñón enfermo; si la nefritis tóxica es muy prolongada, el pronóstico es más incierto, pues puede haber un grado de degeneración muy marcada y el paciente fallecer por uremía

\section{Tuberculosis del uréter, vejiga, uretra,}

Son estas lesiones secundarias a tuberculosis renal, y muestran mejoría después de la extirpación del foco, las le. siones más antiguas se encuentran en el uréter, pero en forma constante lo hace el tercio superior y el medio posteriormente. Como consecuencia de estas lesiones tenemos ectasis parciales, engrosamientos parciales y pérdida de la peristalsis, que más bien puede atribuirse a lesiones tóxicas de la musculatura ureteral. Secundariamente hay acortamiento, y estrechez ureteral, atrayendo el trígono hacia atrás y abajo (cistocopía). Fínalmente la calcíficación del uréter puede convertirlo a éste en un verdadero tubo de pipa, lo que es radiográficamente demostrable.

Las primeras alteraciones de la vejiga aparecen en el trígono, alrededor del orificio ureteral. Al principio sólo hay congestión y edema o tubérculos submucosos. Posteriormente hay ulceraciones serpiginosas superficiales, esclerosis y proliferación de tejido de granulación; el orificio está retraído, 
ensanchado, redondeado rígido, evidenciable a citoscopía. La vejiga puede esclerosarse totalmente y su mucosa tomar un color pálido apizarrado (cistitis crónica atrófica). Secundaria a estas lesiones puede comprometerse el otro riñón por vía ascendente. Las lesiones uretrales son sumamente raras y sólo se presenta en los casos en que el médico está totalmente desarmado.

\section{Síntoma de la tuberculosis renal.}

Los síntomas son los de una pielitis crónica y como en ella, los más bulliciosos son los vesicales. Los principales son: Irritabilidad vesical (74\% al comienzo y $92 \%$ al final): Hematuria $(18.5 \%$ al comienzo y $50 \%$ al final); dolor renal, $22 \%$.

Al iniciarse la enfermedad hay disuria y luego nicturia, a veces hay incontinencia inflamatoria que conduce al diagnóstico de enuresis.

El comienzo de la frecuencia es insidioso, raro es que se presente una hematuria inicial. La polaquiuria no traduce necesariamente una lesión vesical, sino que puede ser la consecuencia de la acción irritante de tuberculotoxinas. Pero si traducen una lesión vesical la incontinencia, el tenesmo, sensación de quemadura, la hematuria terminal. En los últimos períodos hay una gran retracción vesical y el enfermo orina a cada momento.

El dolor renal puede ser cansado, sordo, localizado en los lomos o en el ángulo costovertebral. El pasaje al uréter de coágulos o restos purulentos pueden originar cólicos renales. Sin embargo, en grandes lesiones tuberculosas puede no haber dolor.

La hematuria es un sintoma frecuente, de aparición insidiosa o brusca, de ordinario escasa, pero siempre constante. En los niños. las nefritís y los tumores pueden ocasionar hematurias, pero a veces es también la tuberculosis su agente.

La piuria crónica, macroscópica o miçroscópica deben hacernos investigar la tuberculosis renal. El $2 \%$ de estas piurias en el niño son tuberculosis. Una piuria estéril se considerará como tuberculosis hasta que no se pruebe lo contratio. Colombino ha descrito un signo que lleva gran valcr; consiste en lo siguiente: En un examen de orina tresca se comprueban glóbulos de pus estallados, acompiñados de una hematuria thicroscópica, decimos que es positivo. 
Se citan casos raros de piurias crónicas asépticas aparentemente, pero que son producidas por espirilos (de origen dentario, caries).

Trastornos gastrointestinales repetidos ocutren en el $50 \%$ de los casos en los niños (estitiquez, diarrea, gastritis, etc.): estos trastornos metabólicos hacen que el peso se estacione, que presenten anemia, palidez, astenia, cefalea. Es raro ver que la tuberculosis renal se presente en niños con buen estado de salud aparente. En la tercera parte de los niños con tuberculosis tenal hay fiebre, que tiene estrecha relación con el drenaje urinario.

Es raro que se diagnostique una taberculosis renal por encontrar una masa renal palpable, debido a una fibrolipo. matosis perirrenal intensa, si se trata de un absceso perinefrítico, no sigue los movimientos respiratorios.

\section{Diagnóstico.}

Anamneris. - Antecedentes familiares tuberculosos, contagio tuberculoso, signos urinarios en un niño que vive en ese ambiente o que tiene una lesión tuberculosa en evolución en otro órgano.

Examen físico. - Daremos por descartado un examen completo, especialmente investigando manifestaciones en otras vísceras o ganglios. Es indispensable hacer un tacto rectal y ver si es posible palpar el engrosamiento ureteral. Tiene gran importancia la reacción de tuberculina: un niño con reacciones pesitivas y que no tenga lesiones viscerales o ganglionares visibles radiológicamente y que presente manifestaciones urinarias, debe hacernos pensar y descartar una tuberculosis urogenital. Pero ella no nos hace descarta nna lesión tuberculosa renal si es negativa.

Examen de orina. - Debe ser orina aséptica. La orina en la tuberculosis urinaria es siempre turbia y sin brillo. La albúmina puede ser escasa o abundante. La piuria es cons. tante y a!gunos autores como Beer. Kalisher y Katz han observado que ésta aumenta cuando se realiza una reacción de tubercalina. Si constante es la anterior, la hematuria microscópica lo es más aún. Pueden encontrarse cilindres (granulosos o hialinos). que indican una nefritis tóxica. Si aparpcen cilindros de pus, hay una infección secundaria.

La investigación del bacilo de Koch debe hacerse y la mayoría de los autores concuerdan en que debe encontrarse en el 80 a $90 \%$ de los cases en el examen directo y en $1:$ 
totalidad de los casos en la inoculación al cuy, tiene la inconveniencia de la demora que es de cuatro a seis semanas.

Estimación de la función renal.

El test de la fenoptaleína, la uremia y el nitrógeno no proteico tienen importancia. Raro es que esté alterado, saivo que exista un compromiso bilateral, o una nefritis tóxica muy intensa. El examer de las muestras ureterales indicará las funciones comparativas de los dos riñones.

La citoscopía debe hacerse siempre, pero por la gtan hiperirritabilidad vesical debe hacerse bajo anestesia generai, - raquídea. Las lesiones varían desde una simple congestión y edema del trígono (orificio ureteral del lado enfermo); hasta la formación de úlceras tuberculosas típicas en sacabocados, superficiales, pequeñas, irregulares y por coalescencia se puede formar una gran lesión serpiginosa. En el último estadio, el meato permanece permanentemente abierto, tedondo y ligeramente retraído, debido a la cistitis primeatal y a! acortamiento por retracción esclerótica del uréter. E1 trígono puede estar rechazado hacia atrás y afuera. En casos muy avanzados, la citoscopía es extraordinariamente difícil, pues hay retracción esclerótica, hemorragias, ulceraciones, granulaciones, edema y restos purulentos. A iveces, es necesario recurrir al índigocarmín para poder encontrar los orificios ureterales.

Es indispensable caracterizar ambos uréteres y practicar los test funcionales endoyenosos ( $\mathrm{K}$ de Ambard, etc.) y al misno tiempo, un examen microscópico de ambas otinas e inoculación al cuy. Ya vimos que en la muestra obtenida del fín sano podíamos encontrar cilindros, debido a nefritis tóxica. La cateterización bilateral no ofrece peligro de llevar la contaminación al lado sano, siempre que haya un buen drenaje. (Braash).

\section{Urografía.}

Generalmente el diagnóstico de tnberculosis renal ya se ha hecho cuando recurrimos a ella. Constituye una de las investigaciones más seguras y precisas.

Antes diremos que las lesiones parenquimatosas tuberculosas no conectadas con la peivis no muestran cambios pielográficos.

Precozmente puede haber un ribeteado o franjeamiento de los cálices; y si hay ulceraciones que drenan a la pelvis. 
encontramos sombras urográficas periféricas, el contorno de los cálices son irregulates. Si es un caso más avanzadó, pueden comunicarse estas ulceraciones, dando un pielograma más irregular; lo corriente es encontrar un contorno pélvico irregular, como comido de polilla. Junto a esto hay lesiones asociadas de la pelvis y del uréter debido a los procesos de esclerosis y estrechez. Las imágenes pionefróticas son iguales a las no tuberculosas, se pueden diferenciar sólo si hay depósitos calcáreos (riñón mastic*).

La imagen ureteral es típica, el lumen está irregularmente estrechado, especialmente en el tercio superior, con dilataciones irregulares entre los estrechamientos.

Si no es posible caracterizar el uréter, recurrimos a la pielografía endovenosa, la que en los casos precoces es de poco valor. La eliminación en lado normal puede ser completa, no asi en el afectado en que hay un tetardo. Al mismo tiempo permite apreciar las lesiones pelvio-caliciarias y ureterales ya descritas. Los riñones muy alterados o pionefróticcs pueden no dar eliminación.

\section{Tratamiento de las tuberculosis renales.}

Es una enfermedad progresiva y la autonefrectomía que a veces se produce, sólo pospone la tragedia, pero no la aleja (Campbeli). La nefrectomía debe practicarse una vez establecido. el diagnóstico, siempre que las condiciones del paciente lo permitan: queremos decir que no haya otro foco en actividad; además, debe haber una buena excreción nrinaria en el otro riñón, ya porque éste sea también tuberculoso o porque presente una nefritis tóxica muy intensa que exponga a una muerte por uremia.

Siempre al tratamiento quirúrgico debe seguir el tratamiento sanatorial.

Antes era un problema delicado la cicatrización de la herida atribuída a la infección del muñón ureteral; esto se ha simplificado ahora con el uso de sulfathiazol local.

Si la lesión vesical no ha tenido macho tiempo de evoJución, ésta cesa espontáneamente con ectomía del órgano enfermo; pero en $20 \%$ de los casos la regresión no se hace, quedando los operados con las mismas molestias subjetivas que antes de ser intervenidos;' Esta eistitis se tratará con los métodos corrientes y si na cede despnés de dos años, la daremos como definitiva. 
Algunos autores usan con resultados muy satisfactorios en el post-operatorio la tuberculina. empezando con dosis de $0,000001 \mathrm{mg}$. de $\mathrm{T}$. A. durante 10 a 14 días, suspendiéndola a la menor reacción local o general (Campbell).

\section{Pronóstico.}

Mejora enormemente con la nefrectomía precoz. Si no es intervenida la enfermedad, es progresiva y puede ir a la diseminación. A mayor edad del paciente, mejor es el pronóstico. "Bajo los dos años mueren las dos terceras partes de los niños, aun intervenidos a tiempo".

La mortalidad operatoria es baja, fluctúa entre 0 y $5 \%$ en las más malas estadísticas. Los dos primeros años del post-operatorio son los críticos. Un $20 \%$ de los operados mueren de tuberculosis urogenital; de ahí que el pronostico sea mejor en las mujeres.

Falci encontró que en 25 niños operados de tuberculosis renal, 6 estaban curados después de varios años, 4 permanecían estacionarios, 4 habían empeorado y 11 babian muetto.

Campbell, de 11 casos encuentra lo siguiente:

8 operados viven,

1 falleció, pero era bilateral.

7 no operados, por ser bilaterales, o por compromiss de otro órgano, hath muetto todos.

2 operados de nefrectomía, junto a epidedectomía. hubo recrudescencia al año.

\section{P. O.. - H, R, del Ríg N.: 41/2773}

Antecedentes bereditarios. - - Palire de 47 años, padece de afección cardíaca. Madre de 35 años, con reacción de Kahn negativa; enferma de tuberculosis pulmonat hace 10 ä̈los, sometida a tratamiento con pueumotórax y dadi de alta bace 7 meses, siguiendo actualmente bajo control médico. Das hijos. el otro padece de uta adenopatía hiliar tuberculosa. Un aborto terapético de dos meses. Un tío materno tuberculoso visita esporádicamente la casa. Los abuelos maternos fallecieron de tuberculosis palmonar, en la actual residencia.

Antecedentes personales, - Nació en parto normal, de término: iatendida en la maternidad del Salvador. Pesó $3700 \mathrm{grs}$. Sepadado de su madre al nacer, por estar ellar taberculosa. Atendido en el Seguro Obreto (Servício de Colocación Familiar), hasta la edad de once meses, La alimentación fúé con- 
TUBERCULOSIS RENAI QUTRURGICA EN EL N!N̄̃o. RESUMEN DE 18 CASOS, SEGUN CAMPBELL

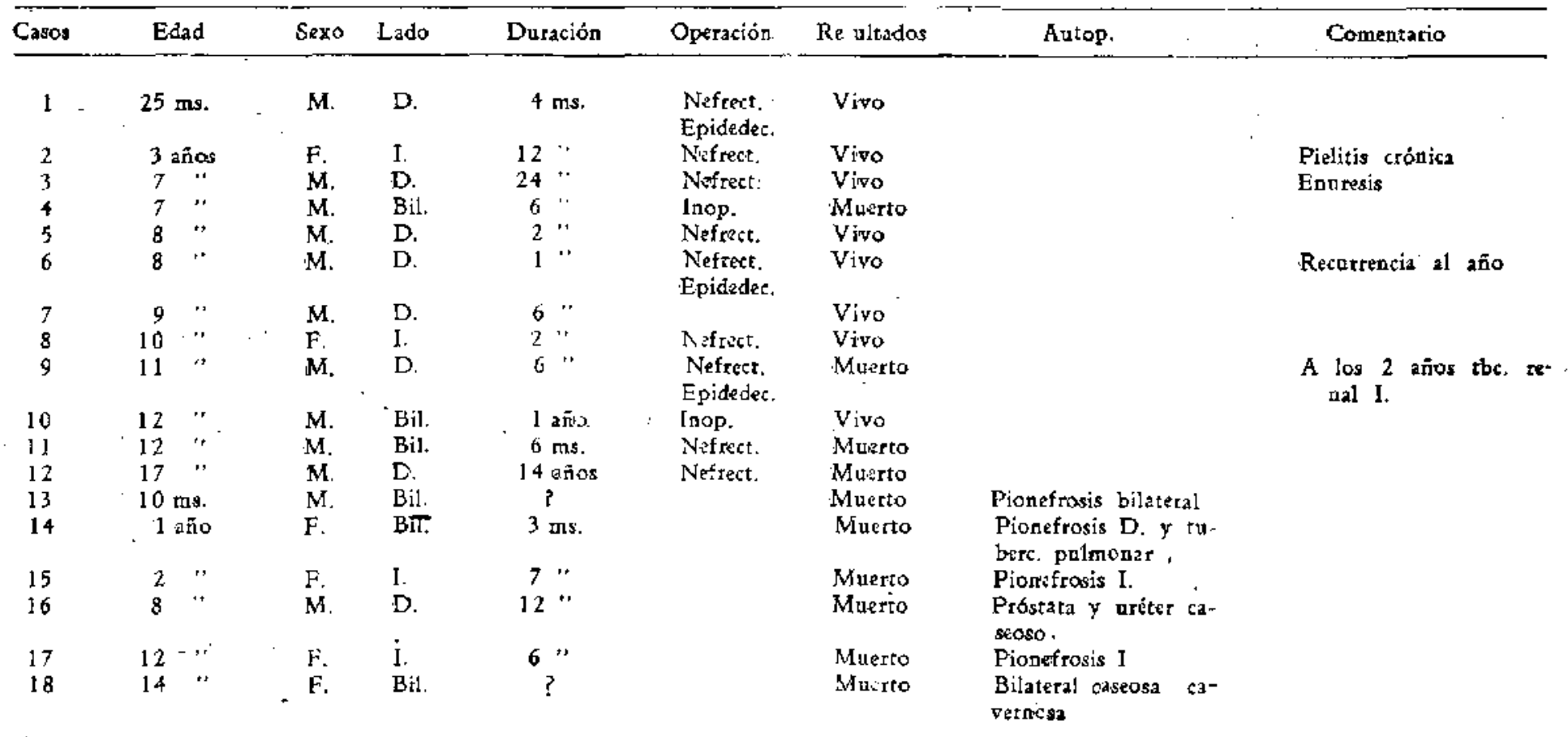

En resumen: doce obsermaciones dinicas y seis post-mortem. 
trolada por pediatras basta la edad de dos años. Post2riormente, al s*r rutiradio del Seguro Obrero, recíbió una alimentación completá.

Enfermedades anteriores. - Bronconeumonia antes del año de edad, atendido en el Hospital de Niños Roberto dtal Rio, dado de alta en bruenas condicicnes. En esta ocasión tuvo un Mantoux negativo. Al año sais meses, el - niño ya vive con su madre y tiene un Mantoux negatio al 1 x 1.000 (verificado en el Seguro Obreroj.

Enfermedad actual. - Desde varios dias arrás, Ia madne lo nota decaido, inapetente, sediento, prezentando vómitos esporádicos y dolores abdomisátes. Aḑemás, el niño estaba intrąquilo, llorós $y$ enflaquecia progresivamente.

Examen de ingroso, - Niñio de dos zanos seis meses, de talla nocmal. Regular estado nutritivo. Posición activa, Temperatura rectal, 38\%. Presión, $85 / 4$ al tonometer. Palidez acentuada de la piel, especialmente a nivel de los pabeliotes auditivos. que muestran un color amarillo-céreo. Tinte de la piel amaciliento-moreno.

Cabeza. - Fontanela anterict certada. Eminencias óseas muy marcadas. Frente prominente. Cara simétrica. Ojos: escleróticas azules con 'algunas manchas apizarradas. Conjuntivas anémicas, vasos ligeramente matcados y sinnocos. Nariz: cotiza con secretión muco puralenta escasa. Buca: encás ligetamente vascularizadas. Faringe roja. Dentadura completa y en buen estado.

Cuello, - Micropoliadenia ervical. Dizcría ingurgitáción yuginlat.

Tórax, - Simétrico. Huadido lateralmentê. Rcrario contal esbozado. Corazón: limites y conos normales. Pulmones; escasos estertores bronquiales en ambos campos.

Abdomen. - Globuloso, pequeña hernia supraumbilical de la línea nedia. Circunferencia abdominal, 46 centimetros. Inspección: Aumento de roicmen en la región del bipocandrio y flanco izquierdo. Palpación: Se toca a ere nivel una tumoración dy, consistencia dura. de superficie regular, de borfe cortante, en el que se apreciarl algunas escotaduras, indoloras, que sigaen los movimientos respiratorios. Esta tumotación se extiende hacia abajo hasta la cresta iliaca y. lateralmenté ocupa el ángulo costovertebral izquierdo y llega hasta das travesa de dedo ge ta linea media; a nivel de la Jínea exilar media. mide 9 -cms. y tiene 11 de ancho. Higado se palpa a nno y medio través.

Región ancegenital. - Micropoliadenia inguinal.

Extrzendades. - Ligrera cianosis de las uñas. Dedos hipoctáticos de ptimer grado.

Sistema nervioso. - Reflejos normales: sensorio normal.

Evolución clínica. - Se le indicó régimen completo y seposo: con ello rajóó notablemente el estado general, y el niño prosentaba buen apetito, aunque algo caprichoso. Diariamente hacia temperatura que llegaba hasta 39 en Las tardes. sin que ella lo afectara grandemente. La tumoración ya chescrita. cambiaba de tamañó $y$ consistencia $y$ aun en ocasiones era dolorcsa $y$ en los últimor dias permanentemente sensible, con carácter tenirente. Simultáneamente 
con la disminución de tamaño del rumor, el tiño hacía bruscas bajas de peso. Al mes y medio de estada en el Hospital se auscultaron en la base del pulmón ziquierdo frotes pleurales, los que pasaton espontáneamente. sin encontrar lesión rádiológica. La diuresis del lactante fluctuaba alnededor de 300 a 550 cc. en las veinticuatro horas. El niño también presentó tajastornos disúricos caracterizados por: dolor a la micción, orina gota a gota, tenesmo vesical. Fote cuadro no mejoró con tratamiento sulfathiazólico.

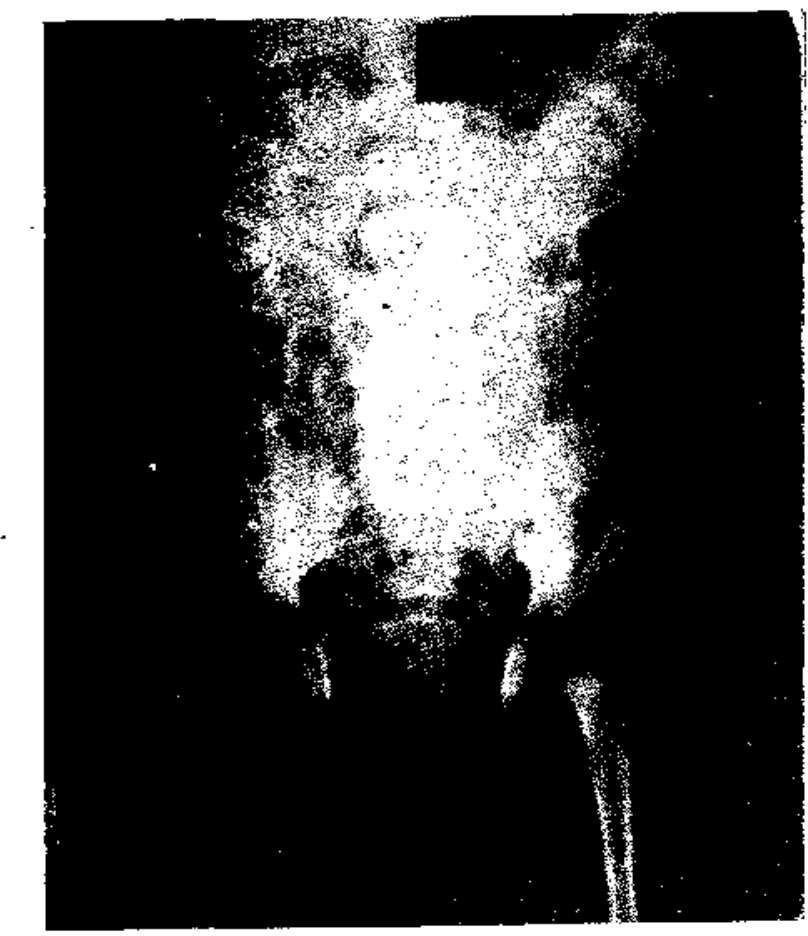

Pielografia.

Erä́menes. - Era un niño ccn tantos antecedentes fámiliares tubetcalosos de shi que esta enfermedad fué la primera que te trató de descartar. Un Mantoux al $1 \times 1,000$ faé intensamente positivo, a contisuación se pidió una radjosiopía y luego una radiografia pulmonar, las que fueron nagativa: Estabancs en presencia de un nin̄o con una tumoración abdominal, que tenía un Mintoux positivo y no presentaba lesión tadiológica en el tóras. En oliso; exámen's realizados, tales como hemograma: se pudo apreciar en él una dis¿reta anamia hipocrómica, leucacitosis moderada $y$ linfocitosis. Y en los axamenes de orina no se encontró absolutamente nada patológico. 
Ante ia posibilidad de una afección esplénica (estenosis de la vena esplénica), se investigó bemorragias ocultas en las deposiciones y se realizó una radiografia de esofago, por medio de contraste, que resultaron tegativa.

Sólo-a los 25 días de ingresar al Hospital, en el octiavo examen de orina, aparece una pinria microscópica y una escasia hematnria, e indicios de albúmina. En exámenes posteriores persistieron estos caracteres, aunque no en en sa totalidad. La investigación del signo de Colombino y del bacilo de Koch en la orina fué negativo. Al constatarse la piuria se le indicó tutatamiento salfamidado. el que no la mejoró en absoluto. En los uroctultivos no se enccontró germen 21 sulal atribuír esta piuria crónica.

Se realizó nna pielografia descendente con prabrodil y el profesor Opa7o. del Hospital Clínico San Vicente, informó categóricamente: riñón mastic tuberculoso izquierdo. so se aprecia lesion radiológica al lado derecho.

Se Jievó a nuestro dactante en consulta al profesor Bisquent. iquien estuvo de acuerdo con la supozición diagnóstica y opinó que se debía intervenir pronto.

Iniciamos la preparación pre-operatoria, con nna serie de transfu-ionss. Fué operado el dia 13 de enero de 1942. El profesor Bisquertt da lla siguiente descripción de la operación: Incisión de Gnyon. Se aprecia inuecriatamente el riñón izquierdo muy aumentado de tamaño, rodeado de gran perinefritis. Se busca un plano de clivaje a tijera, el que se encuentra fácilmente. Nefrectomía. Se aprecian gruesos ganglios del pediculo renal. Se cierra por planẫ. dejanto an drenaje tubular.

La evolución postoperatoria no pudo haber yigto más satisfactoria; el tubo se retiró a los seis đias $y$ h supunación escasa residual se trató con culfatiazol local, a los 12 dias había cicatrizado tozalmente.

Se realizaren los exámenes indispensables para enviarlo a clima; otina, radioscopia de tórax y sedimentación faeton todos normales.

Se trasladó a una playa (Quinteros), donde permanació un año.

Fuś controlado a los 3 meses, encontrandose un nifio en excelente estado general, con baen apetito, $y$ dos kilos más de peso. La ractiografía de tórax fué negatioa, así como la investigación de pioria, bematuria y bacilo de Kocb en la orina, Al año 10 meses, el niño habia aumentado cinco kilos, y los exá. menes practicados también fueron negativos como los anteriores.

Comentario. - Se trata de un lactante de 2 años 6 meses, con antecedentes familiares tuberculosos, que hasta el año 6 meses tiene un Mantoux negativo. Ingresa al Servicio de Lactantes, por presentar decaimiento, enflaquecimiento y palidez; se comprueba en él una tumoración abdominal izquier. da , acompañado de una piuria y hematuria de evolución crónica que no cede a los sulfamidados. Presenta, al mismo 
tiempo, un Mantoux positivo sin lesión clínica ni radiológica en el tórax. quierda.

Se diagnosticó en él hidropionefrosis tuberculosa iz-

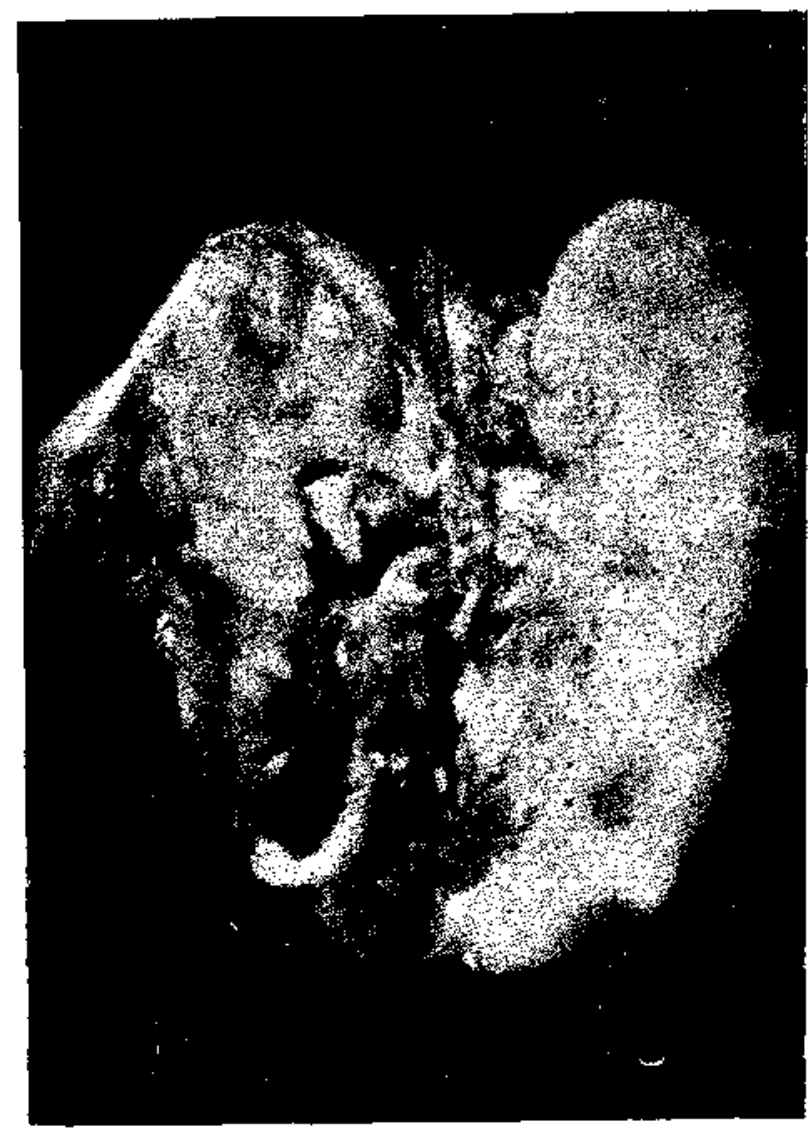

Fotografia del riñón recién extirpado.

Se bizo la ectomía renal y se encontró un riñón tuberculoso típico. Se trataba de un ótgano muy aumentado de tamaño, y que al corte presentaba masas de substancias caseosas, algunas zonas como el mastic. La pelvis renal estaba muy di atada y obstruida casi en su totalidad.

$\mathrm{El}$ informe histo-anátomo-patológico realizado por el doctor Guzmán, dice lo siguiente: En la mayoría de los cor- 
tes del riñón practicados se encuentra un proceso, inflamatorio, difuso generalizado, con gran desarrollo del tejido conjuntivo y atrofia del parénquima renal. En un corte se encontró un nódulo tuberculoso típico, epitelióideo gigantocelular.

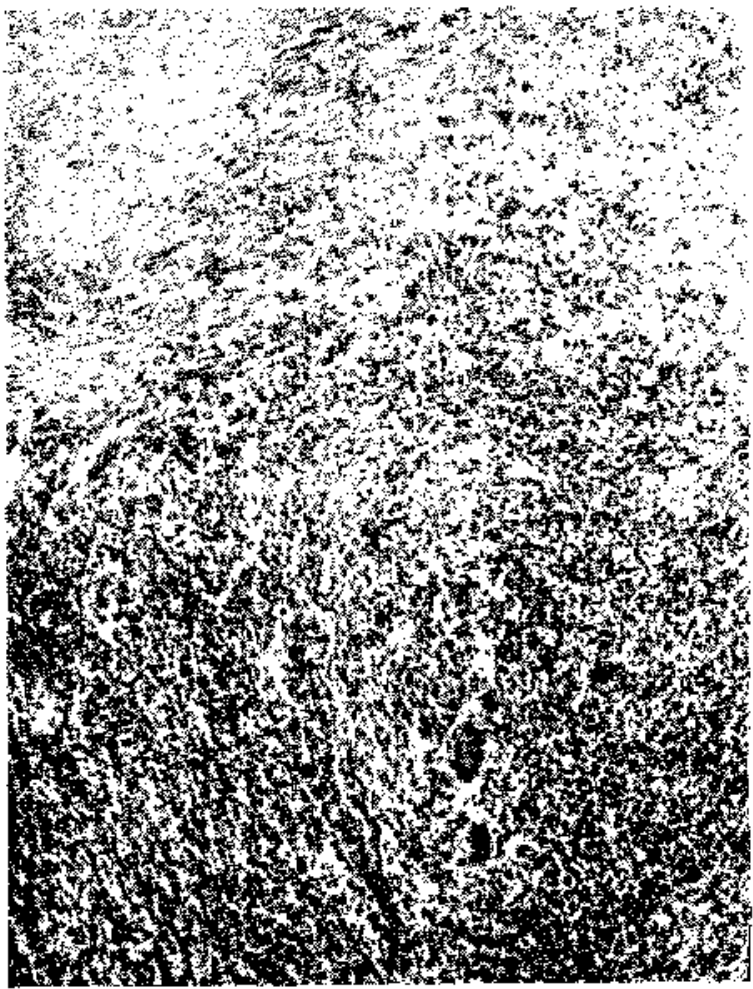

Microfotografía. Corte parébquima renal.

Conclusión: Atrofia renal fibrosa probablemente secundaria. Hidronefrosis. Tuberculosis productiva fibrosa concomitante.

Se acompaña una fotografía de la pieza anatómica, $y$ nna microfotografía de la zona deserita y también una foto. grafía de la placa radiográfica (pielografía. 11 minutos).

A continuación copiamos un resumen de los exámenes practicados. 


\section{ORINA :}

16- IX-42.-Negativo.

20- X-42.-Negativo.

12- XI-42,-Alb-umina indicios, Glóbulos de pns, Hematies,

18- XI-42.-Albúmina no hay. Glóbulo de pas no hay.

2-XII-42.-Albúminas indicios. Glóbuloz de pus.

9-XII-42.- Niegativo.

19-XII-42.-Albúmina 0,20 gr. Glóbules de pus. Hématies Cilindros gràn.

23-XII-42.-Negativo.

14- I-42.-Bacilos de Koch Io se enconitaron,

27-XII-42.-Bacilos de Koch no se encontzaron al examen directo ni a la orina concentrada. Cultivo negativo. Cl. Un. Ut. Prof. Bisquertt.

18. II-42.-Negativo.

27. IV-42.-Negarivo.

UREMIA.0.40 gr. $\times 1,000$.

HEMOGRAMAS:

Henaties Hb. Leucocitos Bas. Eos. Bac. Segim. Linf, Monocitos $20-X-42$ :

$\begin{array}{lllllllll}3.430 .000 & 8,5 \% & 17.900 & 0.5 & 2 & 10 & 31 & 53 & 3, j\end{array}$

$27-X-42$ :

$\begin{array}{lllllllll}3.890 .000 & 8.8 \% & 15.800 & 0.5 & 1 & 22 & 39 & 31 & 5\end{array}$

$5 . \mathrm{II}-42$ :

$\begin{array}{llllllllll}4.390 .000 & 12 & 6 & 17.500 & 0.5 & 3 & 10 & 48 & 31 & 4\end{array}$

Kahr. -Negativo.

Bilirubinemia: $0.17 \mathrm{mlgr}$.

Diámetros globulares Máximo, 12 micrones: mínimo, 4.5 micsones.

Resistencia globuiar: Normal. Con hematies desplasmatizados y lavados.

Reacción de Weber: Negativa.

Reacción de Triboulet: Negativa.

Deposicicnes: Negativa.

Baciloscopia: No se encontraron bacilos de Koch.

Piclografía: Riñón. Mastic, Pref. Opazo.

Radiogtafia carpo: Normal,

Radiografia esófago: Normal.

\section{BIBLIOGRAFIA}

ARON and HIRSH, - Monatschr. f, Kisderb. 1929, 43, 385.

BARNEY and JONES. - Boston M. and Sarg. Juor. 1925, 193, 540.

BEER and HYMAN. - Diseases of the Urinary. tract. in Children. N. Y. I 929

BUMPUS and MEISSER, - - Arch. Int, 1921, 27, 326. 
BUMPUS and THOMPSON. - Serg. Gynec, and Obst. 1928, 66, 791 BRAASCH. - Am. J. Med. Sc, 1920, 156, 8.

BRAASCH and MORSE - J. Urol, 1927, 17, 287.

CAMPBEILL., - J. A. M. A. 1922, 98, 1729 .

CAMPBELLL. - J. Urol. 1933, 30, 245..

CAMPBELL. - Pediatric Utology, vol, 1. Ed. N. York.. Macmillan Company. 1937.

CLARK. - J. Urol, 1934, 31, 193. Lantet, 1932, 2, 511.

ECKEHORN. - Tubercle 1923, 4, 337 .

FALCI. - J, d'Urol, 1925, 20, 301.

FJNKELSTEIN. 一 Jahrb. F. Kinderh, 1896, 43, 148.

FINKELSTELY. - Tratado de las entermedades del lactante. 1940.

GRUBER, - J. Urol. 1930, 23, 161.

HELMHOLZ. - Proc. Mayo Clin. 1936, 11, 231..

HELMHOLZ. - J. Urol. 1934, 31, 173.

HELMHOLZ. - Clinical Pediatries. Appleton, 1930, XX, 138, 1.

HELMHOLZ. - Wiscosin M. J. 1927. 26. 51.

HELMHOLZ and MLLLIKEN. - Am. J. Dis, Chil. 1922. 23. 309.

HEITZ BOYER. - J. Med. Trans. 1922, 11, 178..

HINMAN. - Tr. Am. Assn. Genito Urin Snrg. 1913, 8. 32. 5.

HINMAN, - Am, J. Dis. Cbil, 1919, 17, 305..

IBARRA y STEEGER. — Rev. Cbil. Pẹd. 1937, 979.

J. ISRAEL. - Chirurgie der Niere und des Harnlieten. Leipzig 1925.

KRETSCHMER and HELMHOLZ, -- J. A. M. A. 1920, 73, 1303..

KAPSAMMER, -- Tnberculosis of the Kidney, Wein Brannüller, 1907

LEVY. - Deatsches Arch. f. Klin. Med. 1921, 22. 138.

LEBER. - Dentscb. Med. Wenscbr, 1884, 13, 206.

MAC CARTHY and RIT:TER. 一 Tr. Am. Asn. 1933.

PELS-LEUSDEN. - Qnoted. by Eisendrathand Rolnik, Urology. Pbila, Leppincott, 1928, 703.

POLLACK - Wein. Med. Presse. 1871, 12, 456.

RAFIN. - Encly. Franc. d'Urol. 1914

ROSENOW and MEISSER. - J. A. M. A. 1922, 78, 26, 6..

ROSS. - Pennsylyania. Med. Jour. 1915, 16, 19.651..

THIEMICH. - Jahrb. f. Kindirb. 1910, 72, 243.

THOMAS. - An. J. Suxg. 1930, 10, 308.

VIGNARD and THEVENOOT. - J. d'Urol, 1912, $1,323$.

WILDBOLZ. - Chir, d. Niertuberculose, Stnttegart. Enke 1913

WILDBOLZ. - J. Urol. 1929, 21, 147.

\section{BIBLIOGRAFIA NACIONAL}

BISQUERTT, J. L. - Tesis Universidad de Chile. GONZALEZ MAC OLIVER. - Rev. Chilena de Ped. 829. 10. 1939. 